Official Journal of the Neuroscience Society of Nigeria (NSN)

\title{
Rodent Experimental Model of Konzo: Characterization of Motor Impairment and Neurodegeneration after Cassava Neurotoxicity in the Rat
}

\author{
Lekpa K. David", Chikwuogwo W. Paul ${ }^{1}$, Peace Chigeru1, John H. Martin² \\ ${ }^{1}$ Department of Anatomy, Faculty of Basic Medical Sciences, University of Port Harcourt, Port Harcourt, Nigeria \\ ${ }^{2}$ Department of Molecular, Cellular \& Biomedical Sciences, City University of New York School of Medicine, United States
}

Received:

August 2020

Accepted:

October 2020

\begin{abstract}
Konzo is a motor neuron neurodegenerative disease caused by bitter cassava toxicity that presents as a non-progressive spastic paraparesis. The ability of bitter cassava to produce behavioural and structural changes in adult rat nervous system was examined. Twenty five rats were used for this study, and pruned to 20 after consistent baseline performance was achieved $(n=20$; control=5, cassava chow=15). The rats were switched to a cassava diet for another 5 weeks $(n=15)$. After the cassava consumption period, 5 rats underwent rehabilitation training three times a week and their performance tested once in a week for 5 weeks. Quantitative and qualitative assessments using a reaching movement scale and reach-to-grasp success rate were respectively carried out. Animals subjected to cassava toxicity performed significantly worse than the controls when determining the success rate in a reach-to-grasp experiment (baseline $=69.8 \%$, cassava diet $=21.4 \%$, post-cassava diet $=48.9 \%$ ). The various movement of the rat was analysed using ANOVA and there was significant difference $(p<0.05)$ in the performance. The rats couldn't pronate, grasp, withdraw or open the digits properly when fed with cassava diet. Histology showed neuropathological damages on the motor cortex, less neurons in the motor neuron pool of the spinal cord and disruption of pyramidal layer of the hippocampus when rats were given bitter cassava. Immunofluorescence stain shows motor neurons and numerous choline acetyltransferase (ChAT+) processes and some C-boutons. There are behavioural evidence and neuropathological changes in the motor cortex and ventral horn of the spinal cord that may underlie movement impairments in rats fed with bitter cassava.
\end{abstract}

Key words: Cassava, Konzo, Reaching, Spinal cord, Cortex, Motorneuron, Impairment

\section{INTRODUCTION}

According to Tylleskar et al. (1993) konzo is an upper motor neuron disease which presents as an abrupt onset of different levels of symmetrical, isolated and permanent but non-progressive spastic paraparesis. Konzo as a disease condition was first discovered in Zaire in 1938 (Howlett et. al. 1990). It is a neurological condition that affects the area of the brain that provides posture and movement control of the limbs, resulting in weakness and paralysis. It is produced by toxicity of bitter cassava, a food source in Africa and many other parts of the developing world. The leading cause of konzo has been identified through clinical findings as a combined effect of high cyanide and low sulphur intake from a predominant consumption of insufficiently processed roots of the bitter cassava plant (Manihot esculenta). This is usually the fate of poor and subsistence farmers who depend on eating cassava for survival (Ngudi et. al. 2012).

Correspondence: Lekpa K. David, PhD, Department of Anatomy, College of Health Sciences, University of Port Harcourt, Port Harcourt, Nigeria. lekpa.david@uniport.edu.ng 
Konzo was first described by the Italian physician Trolli, some decades ago, in the former Belgian Congo (now the Democratic Republic of Congo, DRC) (Nzwalo and Cliff 2011). Since then the number of konzo cases reported have greatly multiplied. In Cameroon, Mozambique, Tanzania, Central African Republic and the DRC, outbreaks have been recorded, but proper attention from health authorities was not given. Results from previous outbreaks show that the number of persons affected by konzo is underestimated. Reports presenting more accurate estimates show an alarming number of approximately a million cases in DRC alone in 2000 (Bradbury 2004). According to the Ministry of Health of Mozambique (1984), during the drought period, an outbreak of 1,000 in that country was estimated to have occurred. The local name of konzo in Mozambique is 'mantakassa'. The disease was seen in persons in the remotest areas of the isolated northern province of Nampula. Cultural and religious beliefs also affected the under reporting of konzo cases. In Congo, konzo is the name of a fetish used with traps to catch animals by weakening their legs to prevent escape. As explained by Tylleskar et al. (1993), the appearance of konzo in man was seen as a divine punishment to the persons who used the fetish wrongly.

Konzo affects the motor area of the brain that is known for producing movement and posture of the limbs. The motor area is located in the precentral gyrus on the superolateral surface of the hemisphere and in the anterior part of the paracentral lobule on the medial surface. It corresponds to Brodmann's area 4 and possibly to the part of area 6 that lies in the precentral gyrus. Stimulation of the paracentral lobule produces movement in the upper and lower limbs. Konzo also affects the corticospinal tract, which originates from the motor cortex. This is a descending tract terminating in the spinal cord. Whereas the majority of the tract originates from the motor cortex, some fibres also arise from the premotor area (area 6) and some from somatosensory area (area 3,2,1). From these origins, fibres pass through the corona radiata to enter the internal capsule where they descend in the posterior limb. The cerebral cortex controls voluntary movement through this tract. Interruption of this tract along its course leads to paralysis of the muscle concerned. The neurons that give origin to the fibres of the tract are referred to as upper motor neurons.

Due to the high percentage of poverty in African countries, cassava has been known to be a common food stuff for most households. Cassava can be eaten as whole cassava, grated into chips, processed into cassava flakes or into cassava flour and used in making bread, biscuits etc.

Cassava is a rich source of carbohydrate compared to other carbohydrate-containing foods (e.g. rice and maize). It is also drought tolerant, therefore, it can survive through a season of reduced water supply. Cassava, however, can be classified into bitter and sweet cassava, according to the cyanide content. Cassava is known to contain cyanide with the bitter species containing a higher quantity of cyanide. The cyanogenic glycosides contained in cassava posed a great limitation to the consumption of the root for both human and animals (Sanguanpong et al. 2003; Tonukari 2004).

\section{MATERIALS AND METHODS}

Twenty five adult, female, Wistar rats, weighing between 200 - $250 \mathrm{~g}$ when the experiments began, were used. They were single-housed in wire mesh cages in a room maintained at a temperature of approximately $22^{\circ} \mathrm{C}$ and on a 12-h light/dark cycle, with lights on at 07:30 $\mathrm{h}$ and with water available ad libitum.

Rats were excluded from the study $(n=5)$ because they could not learn to perform the reaching task within a specified period of five weeks, after consistent baseline performance was achieved $(n=20$; control $=5$, cassava chow $=15)$. The rats were switched to a cassava diet for another 5 weeks $(n=15)$. Four more animals dropped out of the study because they failed to consistently perform the task, and 5 animals died, presumably because of cassava toxicity; no death was recorded among rats given rat chow only. After the cassava consumption period, 5 rats underwent rehabilitation training three times a week and their performance tested once in a week, during a rehabilitation session of 5 weeks.

\section{Cassava Preparation for Consumption by Rats}

For development of the model, cassava was prepared as follows. Fresh cassava roots were peeled to expose the white inner layer. The cassava was then diced into smaller sizes like chips. The chipsized cassava was sun-dried for 3 consecutive days. Afterwards, the cassava was ground into a powdery form that was used to make the cassava chow. The cassava chow was administered to the rats for 5 weeks.

\section{Behavioural Assessment}

Animal were trained in a skilled motor reaching task and followed from baseline performance before cassava consumption to the final phase of rehabilitation. They were made to reach-to-grasp a prescribed number of pellets on a pedestal. This behaviour made use of the upper limb in reaching to grasp. Changes in the success rate and efficiency in the process of acquiring the food was used as an indication to show the effect of cassava toxicity on the corticospinal tract. 
Rats were placed on a food restriction for about 2 weeks before training and testing to reduce their body weight to $80 \%$ of baseline. All procedures were approved by the University of Port Harcourt Animal Care Committee.

Animals were trained daily for 5 weeks to achieve baseline performance. Once animals were trained to perform the task and behavioural data collection was completed for the control period, they were placed on a cassava-only diet for 5 weeks. After starting the cassava diet, animals were tested once a week.

In order to properly perform the task, the animals were placed on food restriction to enhance their motivation. This was accomplished by restricting the amount of food the animals received. To minimize stress to the animals, the least restrictive approach was used. Initially, the aim was to achieve up to $85 \%$ of the baseline body weight and used coco pops that are flavoured to encourage reaching (i.e., highly preferred food). We ensured that the least weight reduction necessary to perform the task was achieved and breaks from the restricted food schedule was obeyed for animals that lost weight less than $70 \%$ of body weight. Moreover, during the initial shaping period we eliminated animals from the study that were slow to perform the task. This is in line with both Institutional Animal Care and Use Committees (IACUC) documents consulted (Emory University and University of Pennsylvania, U Penn, USA).

At the start of food restriction, we determined each animal's normal body weight (daily weight for two or more consecutive days). Next, we restricted food to $70 \%$ needed to maintain body weight. The use of $70 \%$ at the start is recommended by both IACUC documents consulted (Emory University and U Penn, USA). We used the following guidelines: rats were given $86 \mathrm{~g}$ of food per kilogram body weight per day (e.g., rats weighing between 200-240 g), was given $23 \mathrm{~g}$ of food per day (U.S.E.P.A. 1988). Weight reduction up to $85 \%$ baseline weight was achieved over a period of one week.

\section{Apparatus}

The testing apparatus (Fig. 1) was custom-made of Plexiglas, approximately $20 \mathrm{~cm}$ long, $8 \mathrm{~cm}$ wide and $20 \mathrm{~cm}$ high. Animals reached through a vertical slit at the front of the cage. A $0.2 \mathrm{~cm}$ thick plastic shelf was mounted approximately $1 \mathrm{~cm}$ from the floor on the front of the testing enclosure. The mechanics of a rat reaching for food are such that food pellet targeted by the rat that are contralateral to the reaching limb are more easily obtained than ipsilateral food targets. Thus, by placing the food to one side or the other, we could elicit reaches from either forelimb, in a controlled manner. The protocol of Whishaw and Pellis (1990) and Ballerman et al. (2001) with minor modifications was followed.

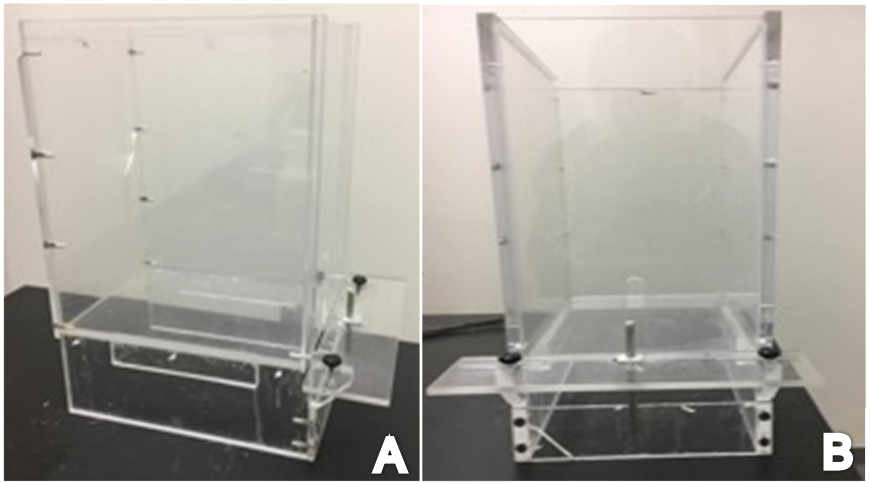

Fig. 1: Reaching apparatus $(A)$ side view $(B)$ front view

\section{Video Recording}

Video recording was done using a camcorder with shutter speed set at $1 / 4000 \mathrm{~s}$ at frame rate. The tapes were replayed for analysis using a recorder with a frame-by frame (i.e., $30 \mathrm{~Hz}$ ) option.

\section{Movement Rating}

A qualitative evaluation of reach-to-grasp movements was performed using a reaching rating scale modified from earlier descriptions (Miklyaeva and Whishaw 1996; Mckenna and Whishaw 1999). The following six movements, which comprise the entire behaviour, were rated:

1. Advance - the head is raised, the elbow is adducted to the body midline, and the forelimb moves through the aperture towards the food;

2. Digits open - the digits are opened and spread as the limb advances;

3. Pronation - the limb is pronated above the target and moves down onto the food with the digits spread to palpate the area of the pellet;

4. Grasp - the limb remains relatively still while the digits are closed. During the grasp, the paw may be supinated to adjust and grasp the piece of food;

5. Withdrawal - the paw withdraws from the shelf, firmly holding the piece of food;

6. Release - the food is adjusted so that it is grasped by both paws, so that one end can be inserted into the mouth for chewing (Whishaw and Coles 1996). Each movement was rated on a 0 - 2 point scale. A score of 2 was given, if the movement was normal (as compared with typical movements of a control rat). A score of 1 was given, if the movement was recognizable, but was abnormal, usually being assisted by another body part. A score of 0 was given, if the movement was absent and replaced by another body movement (Ballerman et al. 2001).

Qualitative Assessment of Movement before and after Feeding Rat with Cassava

In addition to quantifying changes in success, we used high-speed recordings to observe changes in 
the structure of the reaching and grasping movements.

\section{Tissue Processing}

The rats were anaesthetized and euthanized on the last day of the experiment, then fixed through transcardiac perfusion using $0.9 \%$ saline, and then $4 \%$ paraformaldehyde (PFA) solution. The brains were extracted, post-fixed overnight and then transferred to $30 \%$ sucrose for $2-3$ days before embedded in paraffin. Coronal sections of the brain and transverse section of the spinal cord were made on a microtome (Hyrax S50 Zeiss, Germany) at $7 \mu \mathrm{m}$. All sections were taken and organized into 20 parallel series for histological and immunohistochemical staining.

\section{Nissl Staining Procedures}

The sections were first brought to distilled water and then stained with cresyl violet aqueous solution $(0.5 \%)$ for $5 \mathrm{~min}$. They were later rinsed in two changes of distilled water and $90 \%$ alcohol for $30 \mathrm{sec}$ respectively. Next, they were rinsed for $30 \mathrm{sec}$ in absolute alcohol and in xylene for $1 \mathrm{~min}$. The slides were rinsed for $2 \mathrm{~min}$ in equal quantities of Canada balsam xylene mixture. Then rinsed in two changes of absolute alcohol and followed by xylene. Finally, they were mounted in mounted in Canada balsam for microscopy.

\section{Immunofluorescence Staining}

The brain sections were permeabilized for $20 \mathrm{~min}$ with PBS containing $0.3 \%$ Triton X-100 (SigmaAldrich), then blocked for unspecific binding with $5 \%$ goat serum for $30 \mathrm{~min}$ as previously reported (Wang et al. 2016). Goat anti-choline acetyltransferase (ChAT) primary antibody (1:100 diluted in PBS; Merck Millipore; AB144P) was then applied and left to incubate for $48 \mathrm{~h}$ at $4{ }^{\circ} \mathrm{C}$. Slides were washed in PBS ( $3 \times 5 \mathrm{~min})$ and Alexa Fluor 594 donkey anti-goat IgG (1:500; Life Technologies, A-11058) secondary antibody applied for $2 \mathrm{~h}$ at $20^{\circ} \mathrm{C}$ room temperature. Following PBS washes, blocking solution was reapplied followed by mouse anti-neuronal nuclei (NeuN) primary antibody, clone A60 (1:100; Merck Millipore; MAB377) for $2 \mathrm{~h}$ at room temperature. After PBS washes, Alexa Fluor 488 donkey anti-mouse IgG (1:500; Life Technologies, A21202) secondary antibody was applied for $2 \mathrm{~h}$ at room temperature. Following PBS washes, DAPI nuclei stain $(1: 1,000$; Life Technologies, D1306) was applied for $10 \mathrm{~min}$. After final PBS washes, coverslips were placed on slides using Mowoil mounting media. Micrographs were taken using a high resolution Zeiss 880 Airyscan confocal microscope imaging.

\section{Statistical Analysis}

GraphPad prism 8, version 8.1.2 (227) was used to analyse the success rate and movement rating of the animals. ANOVA and t-test was carried out to compare the various periods (baseline rats, cassava fed rat sand post-cassava period) of this experiment. Rat movements and trajectory were analyse using a tracker software (Video Analysis and Modeling tool, version 4.8) and the velocity of movement of the rat was calculated.

\section{RESULTS}

\section{Weight Assessment of Animals}

There was significant difference among means $(p<0.05)$ between baseline, rat fed with cassava diet and post cassava rat $(R$ squared $=0.9221)$. There was weight drop in the animals during the cassava consumption period. During the third and fourth weeks, three rats died. One died on day 21 and two died on day 25 and 27 . We recorded two deaths again on day 40 and 45 . Results showed that animals died progressively after 21 days of eating bitter cassava. By 45 days, 5 animals out of the 20 animals fed with cassava died due to prolong eating of bitter cassava, although the amount of cyanide in the bitter cassava given to the animals was not determined in this study. The survival period or safe-range was only considered. We observed swollen limbs in the rats that survived. Swollen limbs were observed in the group of rats fed with cassava indicating some level of impairment.

To further develop the animal model, systematic examination of the effects of reducing the amount of cassava consumption during the 5 -week period was carried out in order to obtain animals with motor impairment but do not develop swollen limbs.

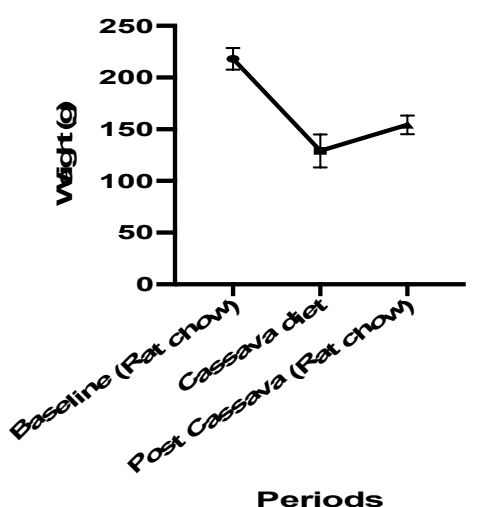

Fig. 2: Average weight of the animals during the 3 period of the experiment

\section{Effect of Cassava Diet on Reaching Performance}

Twenty rats were fed cassava for 5 weeks. Between the 3 rd - 5th weeks, we lost 5 rats probably due to the toxicity of the cassava, and four dropped out of the study as a result of poor learning ability. Rats were trained to learn the skill for 5 weeks. Upon learning the skill with an average success rate above $60 \%$, the baseline data were obtained for each week 
(3 times per week) for five weeks. The average baseline data was $69.8 \%$ success rate. The rats were then fed cassava for a week without training and testing followed by 5 weeks training, testing and evaluation. The average success rate dropped from $69.8 \%$ (baseline) to $21.4 \%$ in 5 weeks. We stopped
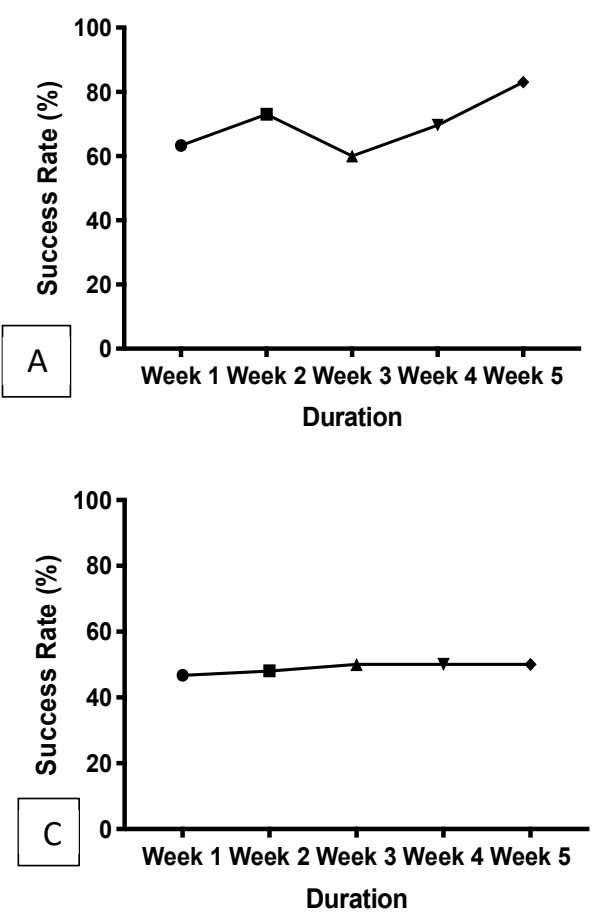

Fig. 3: Success rate scores (A) Baseline success rate across different period.

giving cassava chow to the rat and switched back to normal rat feed for another 5 weeks. During this period, we trained the rats 3 times in a week and test if there was any change in impairment. We observed progressive recovery with slight increase post cassava average success rate of $48.9 \%$ (Fig.3C)

\section{Over-reaching and Under-reaching Movements in Rats}

Fig $4 a$ showed rat trained to perform reach-to-grasp task. The movement pattern was accurate and precise as rat reached to grasp a piece of pellet. The rat aimed at the food pellet with precision indicating that there was no impairment. When the rats were fed with cassava diet, the rat failed to supinate the paw which made the rat used the second paw to support, with the mouth coming in contact with the outer surface of the wrist (Fig. 4b). The paw became weak and the food pellet dropped occasionally. The rats did not aim properly by adducting the limb to the midline leading to overreaching. The advance movement was typically short, and the rat did not fully pronate or supinate the paw to grasp and withdraw the pellet. The rats used the second paw to support the preferred paw during grasping and withdrawal of the single pellet to the mouth. This supporting behaviour was not seen in the rats before cassava was given to them. It was observed that during reaching, the second paw was on the floor, the rat only used one paw to reach and grasp the pellet to the mouth. We also observed under-reaching in the rat fed with cassava. The rats found it difficult to raise the limb to the $3 \mathrm{~cm}$ height of the platform where the food pellet

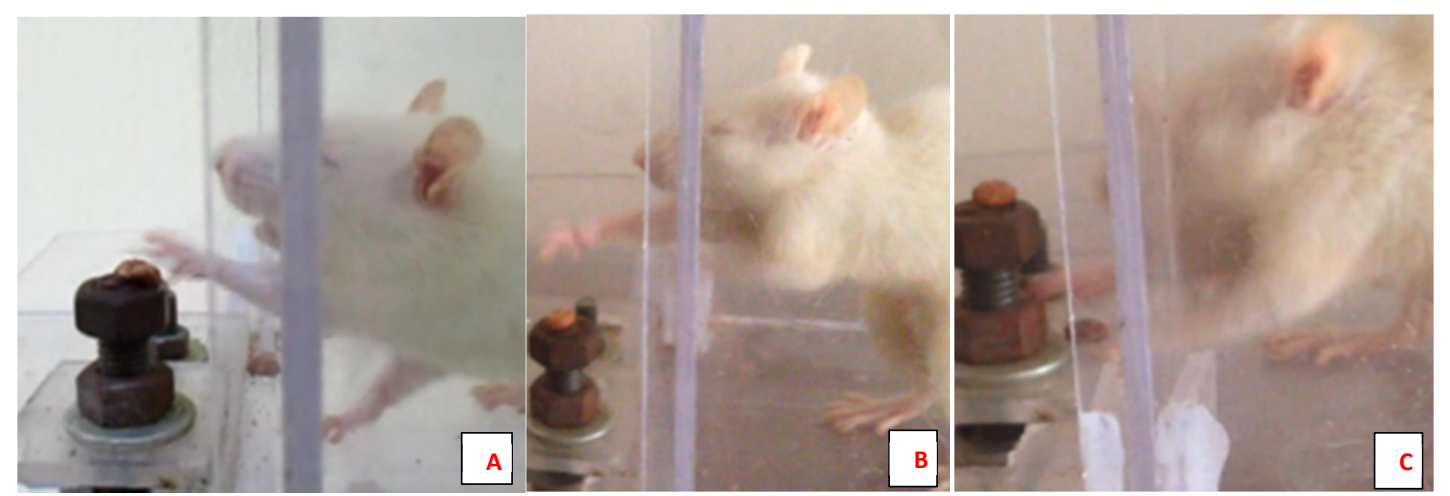

Fig. 4: Over-reaching and under-reaching observed in impaired rat: $(A)$ Rat trained to reach with precision and accuracy before given cassava chow (B) Rat showing overreaching when given cassava chow (C) Rat showing under reaching when given cassava chow 


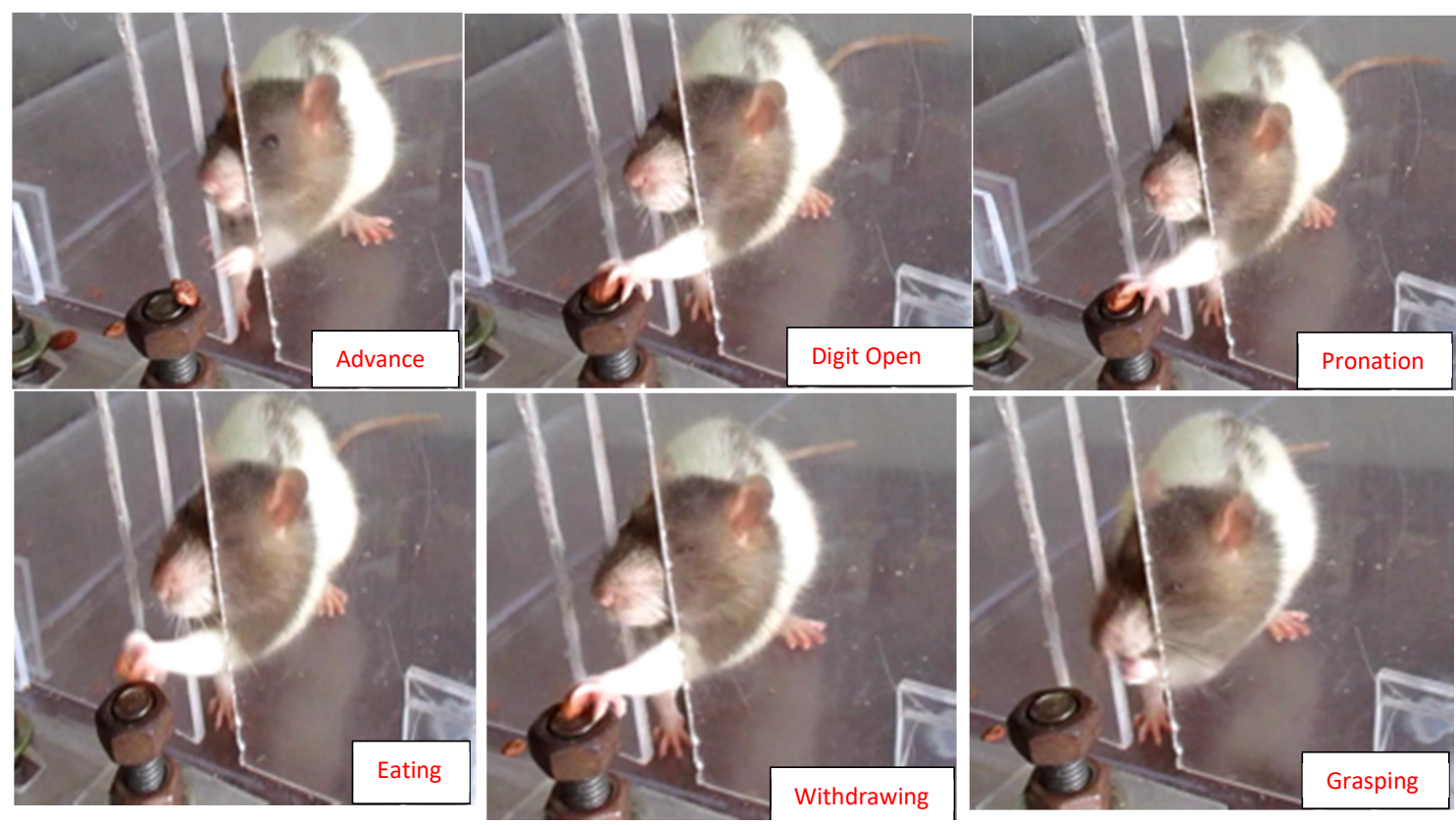

Fig. 5: Qualitative assessment of paw and digit movement made by control rat using reaching movement scale

was placed.

Qualitative Assessment of Paw and Digit Movements in Rat Fed with Rat Chow (Control)

Fig. 5 shows a qualitative testing of the control animals with progressive movement of the paw in grasping the pellet and then eating. There was no sign of impairment since the rats aimed directly at the pellet in the precise direction; reaching for the pellet, grasping, withdrawing its paw and eating the pellet. The animal was able to carry out these movements without support from the non-reaching paw or any other body part. It rested its non-reaching paw on the ground showing that its paws were not weak and needing any support. It exhibited no difficulty in lifting up its limb, since it was not exposed to the cassava diet. There was normal pronation of the paw while grasping the pellet and supination while withdrawing

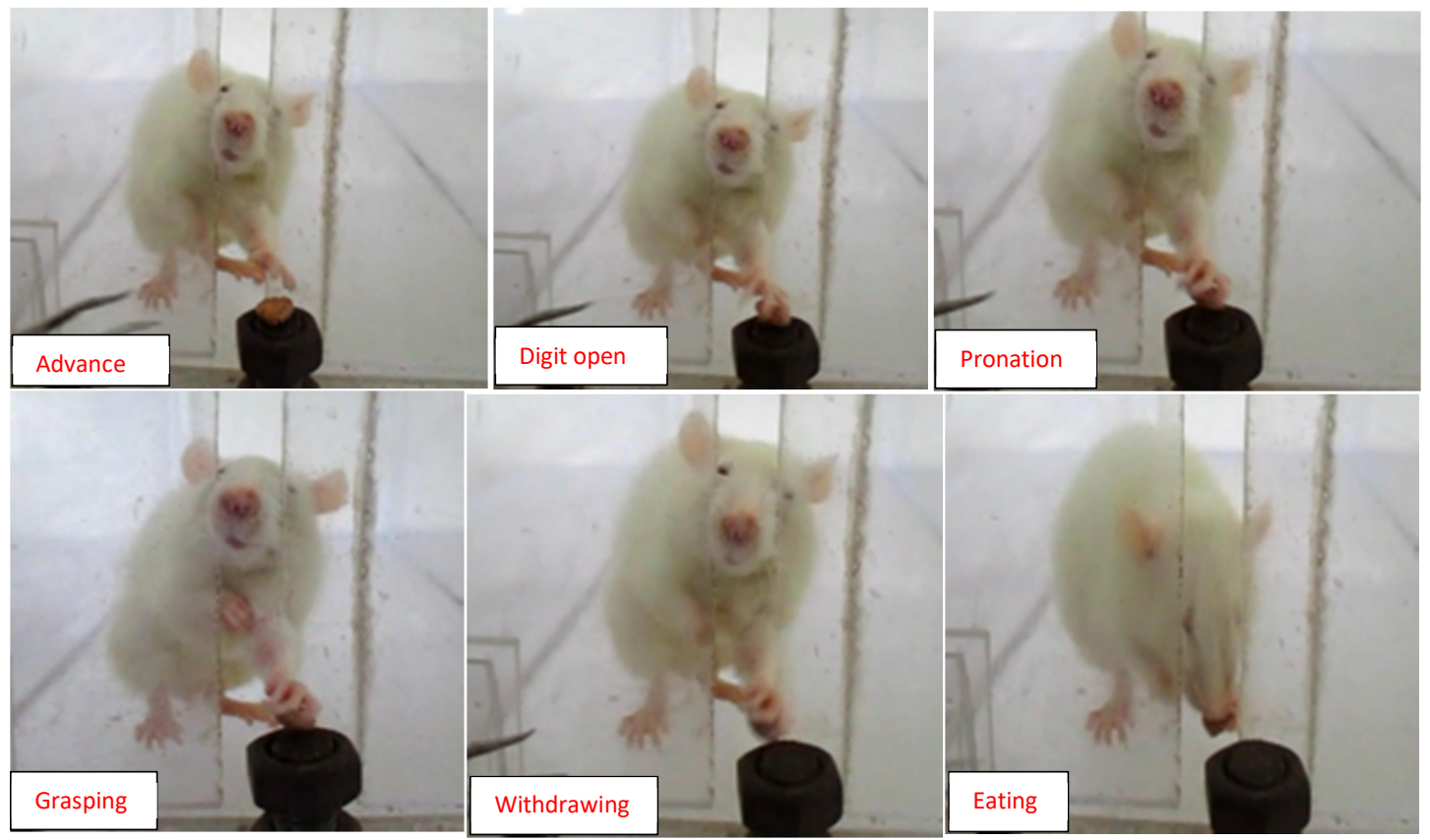

Fig. 6: Qualitative assessment of reaching movement using reaching movement scale for rats given cassava chow. 
its paw. The animal also did not exhibit any form of weakness during the analysis.

Qualitative Assessment of Paw and Digit Movements in Rats fed with Cassava Diet

Although the animals remained motivated to reach, after 5 weeks of eating cassava, the rat failed to supinate the paw which results in adduction of the paw, with the mouth coming in contact with the outer surface of the wrist/paw (Fig, 6). Forelimb supination is a behaviour strongly dependent on corticospinal paw and eat the food in a semi-sitting posture but used a slight compensatory head tilt to retrieve the food pellet (Fig. 7). The deficit in supination was not completely restored however, but a careful observation of the movements of the rats after rehabilitation showed that they were clumsy and that they also continued to have a slight impairment in supination that was sufficient to contribute to impaired performance. The head was turned in the direction of the paw to retrieve the food pellet to help compensate for the impairment.

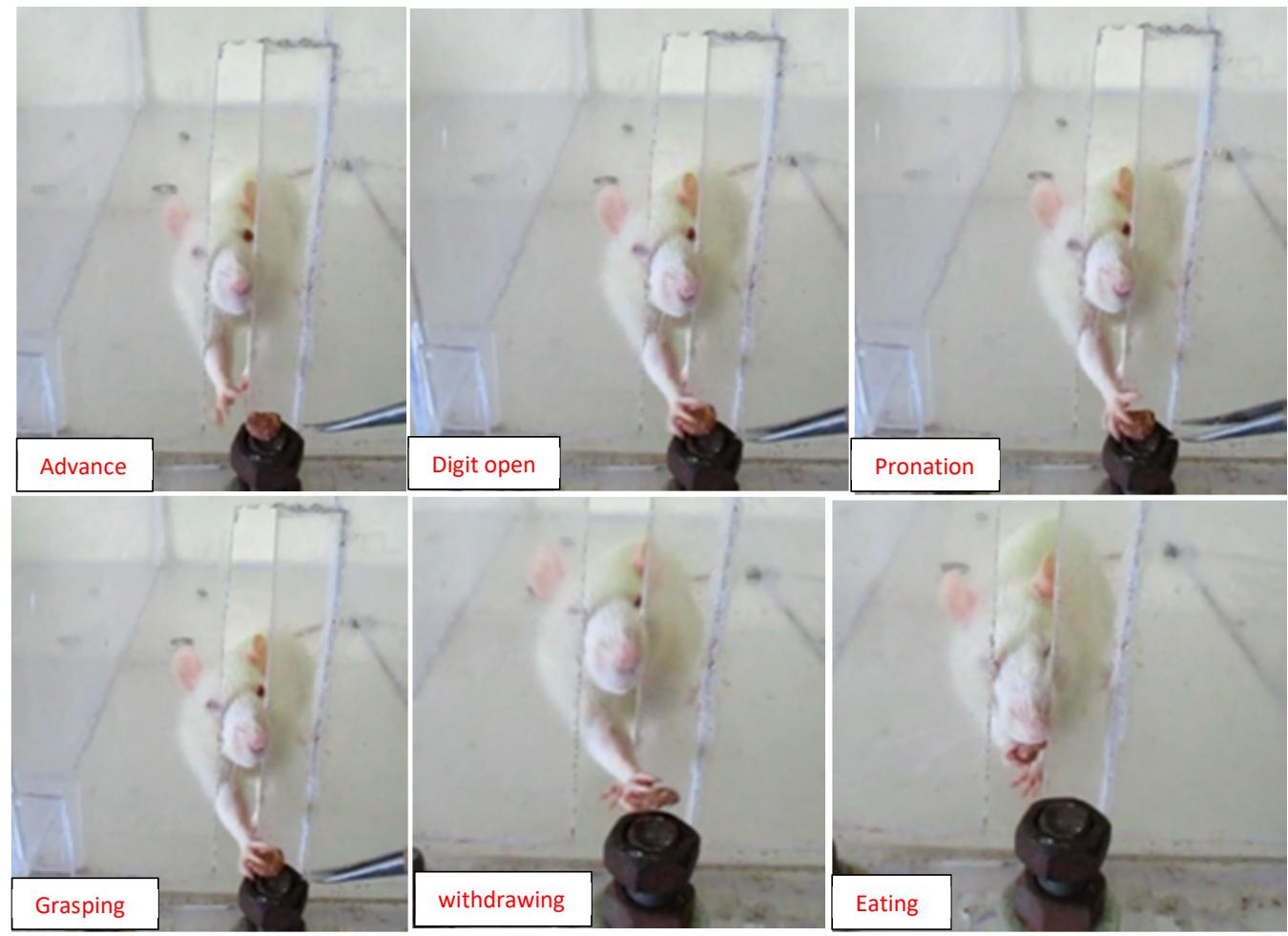

Fig. 7: Qualitative assessment of reaching movement using reaching movement scale for post-cassava rats

system control. The paw becomes weak and the food pellet was dropped. The rats did not aim the limb by adducting the elbow to the midline, the advance was typically short, and the rats did not fully pronate the paw or supinate the paw to grasp and withdraw. The rats often used the non-reaching paw to support the preferred/reaching paw during grasping and withdrawal of the coco pops single pellet in order to get it to the mouth (Fig. 6). This behaviour was not observed in the control rats, where the non-reaching paw was rested on the floor and the rat uses only one paw to reach and grasp the food to the mouth in the control group (Fig. 5)

\section{Qualitative Assessment of Paw and Digit Movements of Rats Fed with Rat Chow after Cassava Diet (Post Cassava)}

After cessation of cassava diet during the rehabilitation period, the rat were able to supinate the
Table 1: A qualitative evaluation of reaching movements

\begin{tabular}{llll}
\hline Movement & Baseline & Cassava & $\begin{array}{l}\text { Post- } \\
\text { cassava }\end{array}$ \\
\hline Advance & 2.0 & 0.0 & 1.0 \\
Digit open & 2.0 & 1.0 & 1.0 \\
Pronation & 2.0 & 1.0 & 1.0 \\
Grasping & 2.0 & 1.0 & 1.8 \\
Withdrawing & 2.0 & 1.0 & 1.8 \\
Eating & 2.0 & 1.0 & 1.7 \\
Total & $\mathbf{1 2 . 0}$ & $\mathbf{5 . 0}$ & $\mathbf{8 . 3}$ \\
\hline
\end{tabular}

\section{Reaching Movement Rating}

The various movements of the rat was analysed using ANOVA and there was significant difference $(p<0.05)$ in the performance. The rat couldn't pronate, grasp, withdraw or open the digits properly when given cassava chow. Although some amount of 
movement was restored post cassava period, we saw signs of recovery after weeks of training and rehabilitation, but not as observed before cassava was given (Fig. 8 and Table 1).

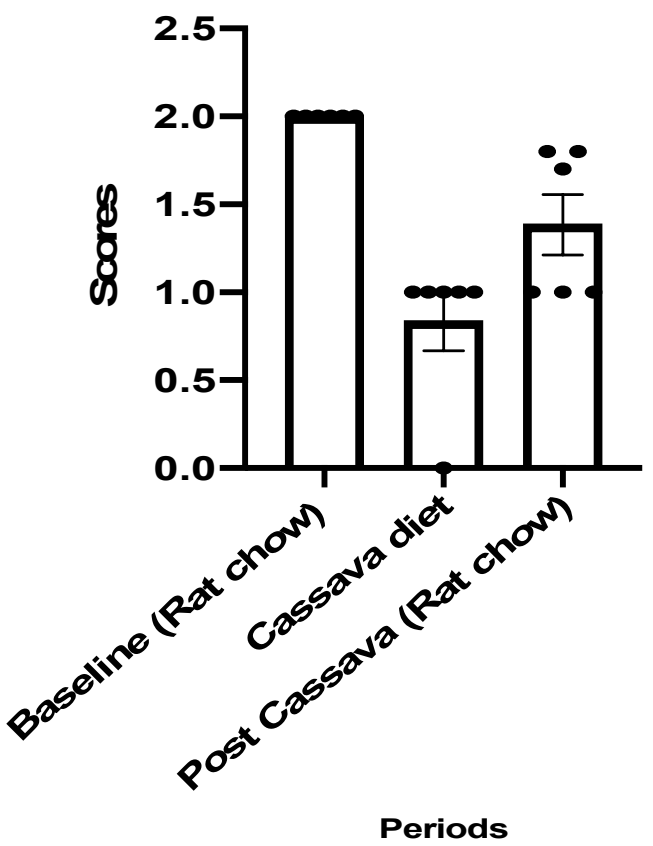

Fig. 8: The average score of the 6 components of the movement for the baseline, cassava diet and post cassava periods.

\section{Trajectory of Movement Pattern in Rat}

This shows different movement trajectories observed in rats using the video analysis software (Tracker, Douglas Brown 2017 version 4.11.0). The baseline period (A) shows smooth and relatively straight trajectories from the floor of the apparatus to the target pellet on the pedestal. The rats at this phase of the experiment made just one attempt before getting to the pellet. Grasping and aiming error was not observed in this group. The rats fed with cassava shows uncoordinated movement (Fig. 9B), with variable trajectories from trial-to-trial. The rats made several attempts before getting the pellet. This is an indication of aiming impairment. We also observed grasping impairment as the rats fed with cassava diet struggle to grasp and retrieve the pellet to the mouth. In most cases this was possible only with support of the second paw (Fig. 6). The post cassava rats showed some level of coordinated movement (Fig. 9C) when compared to the rats fed with cassava (Fig. 9B), but not as precise and coordinated as the baseline (Fig. 9A). The rats were still supporting the preferred paw when making reaching attempt indicating some level of impairment in the rats. The rats also demonstrated some level of over-reaching and under-reaching as seen in the pattern of movements (Fig 9C).

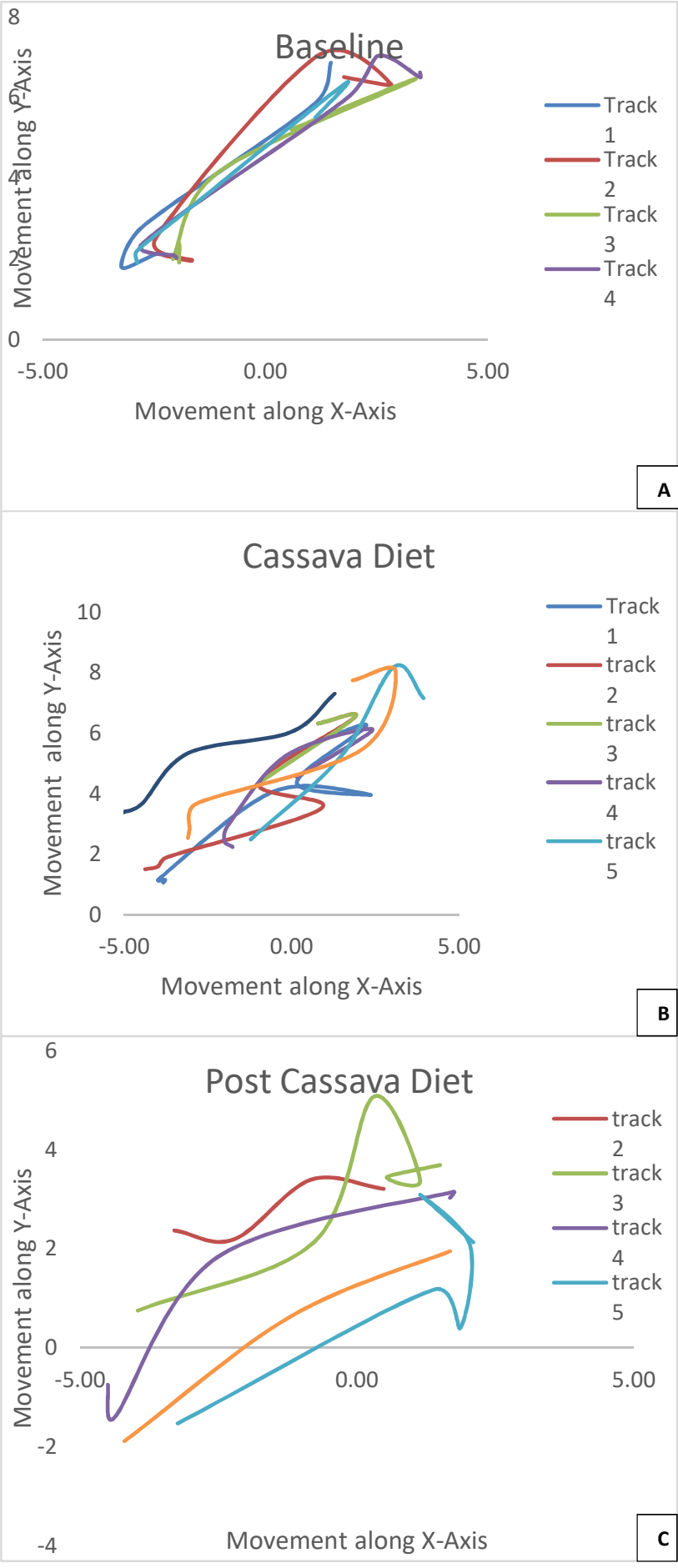

Fig. 9: Trajectory of (A) Baseline (B) Cassava diet (C) Post cassava

\section{Velocity of Paw Trajectory Movement}

We observed a low momentum of the cassava rat paw to reach the pellet at a height of $3 \mathrm{~cm}$ above ground level of the apparatus. Before the rats got to the pellet from the ground level, they made several steps and each point in Figure 10 shows the steps. 
The rats made several attempts before getting to the target and the momentum increasing along the way. The cassava rats couldn't abduct properly at the gleno-humeral joint thereby supporting with the other paw to aid performance (Fig. 10B). It was observed that the velocity of the paw was low when measured using the trajectory tracker program (Douglas Brown tracker 4.11.0). The average velocity for the cassava rats was $(8.89 \pm 1.33 \mathrm{~m} / \mathrm{sec})$ at an average time of $(14.36 \pm 1.60 \mathrm{sec})$. When compared with the baseline average velocity $(8.25 \pm 1.45 \mathrm{~m} / \mathrm{sec})$ at an average time of $(37.61 \pm 1.96 \mathrm{sec})$ and post-cassava average velocity of $(10.25 \pm 1.61 \mathrm{~m} / \mathrm{sec})$ at an average time of $(23.72 \pm 2.11 \mathrm{sec})$, the velocity was low for the cassava animals. This shows that the rats are impaired. The velocity data was computed using the tracker software (Douglas Brown 2017 version 4.11.0) to track the trajectory movement of the rat when performing the task.

Looking at the control rat, the average velocity was higher than the experimental rats, and the momentum at take-off was also higher. This indicates Ithat there was no impairment in this rat.. From the result derived from the tracker, it was observed that the post cassava diet group had the highest velocity of $10.25 \pm 1.61 \mathrm{~m} / \mathrm{sec}$, which decreased as the animal creached the target.

Histological Changes in Rats with Konzo Disease The control micrographs (Fig. 11a) presented neurons with the nucleus closely packed and free of vacuolations. There were numerous neurons as well. The konzo group had less neurons with the presence of nuclear vacuolations (pore spaces) of varying sizes (Fig.11b), The number of neurons in the konzo group were less.

In Figure 12, the ventral horn of spinal cord with Nissl stain showed more motor neurons in the neuronal pool when compared with konzo group with fewer motor neurons in the pool. The sizes of neurons in the konzo group (Fig.12b) were reduced when compared to the control (Fig.12A), which had much bigger neurons. The histology of the hippocampus was observed as well to see if there were any neurodegenerative changes that may affect memory of the acquired skilled movement.

To further see the neuropathological changes in rats that consumed cassava for 5 weeks, immunofluorescence showed motor neurons and numerous ChAT+ processes and some C-boutons (bright puncta of label) (Fig. 14).

\section{DISCUSSION}

Our study demonstrated that Konzo may occur as a result of consumption of bitter cassava in rat. The present study showed that rats' reached for food targets when properly motivated and with the aid of

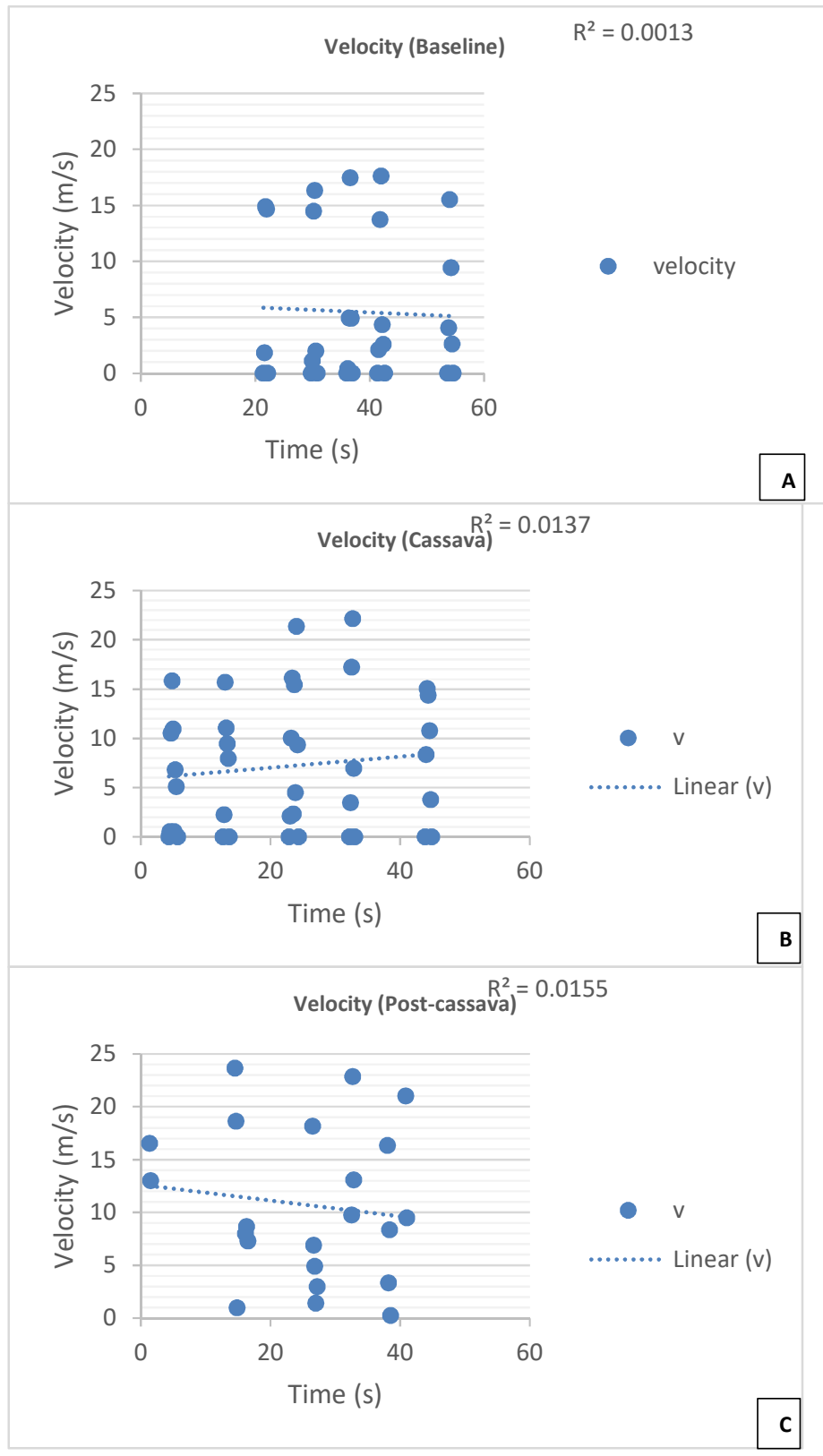

Fig 10: Velocity scattered graph of (A) Baseline (B) Cassava diet (C) Post-cassava

their sense of olfaction. Similar observation was reported by Hermer-Vaquez et al. (2007). Animals fed on low protein diet usually stopped growing, lose weight, and sometimes adapt to the diet at a slow rate (Dollet et al. 1985; Okitolonda et al. 1988). Our study agreed with this statement because cassava is low in protein and we observed weight drop in the *rats fed with cassava only during our experiment. According to Farr and Whishaw (2002), animals had higher reaching scores before lesion occurred. This was observed in our study as the reaching scores of the animal after cassava consumption decreased when compared with the baseline scores. This is an 
indication that bitter cassava may have damaged the motor neuron in the corticospinal tract, hence, affecting the performance of the animals. Previous studies have demonstrated that after a motor cortex injury, a rat still retrieved food by reaching out with the affected forelimb, but the success rate was reduced (Kolb et al. 2000; Whishaw 2000; Biernaskie and Corbett 2011. They also observed that most of the movement components of the reach are abnormal and that compensatory body movements provided the rotatory movements to assist pronation and supination. Our observation is in accordance with those of previous studies that stated how compensatory body movements provided the rotatory movements to assist pronation and supination after a motor cortex injury in rat (Whishaw 2000; Kolb et al. 2000; Biernaskie and Corbett 2011). In the work of
Farr and Whishaw (2002), they compared control and stroke induced animals and observed that although the digits seemed to align with the body in both animals, the stroke animals achieved alignment in part by using body rotation.

The control animals were able to advance the paw from the 'aiming' position directly through the slot towards the food, whereas the stroke animals directed the paw through the slot diagonally. A similar result was observed in the present study. There were impairments in the limbs of the rats fed with cassava as the movement were altered even when the rats successfully obtained the food. The paw and elbow were not aligned to the aiming position along the midline of the body, but rather moved diagonally towards the food. Several research works have been done and reported on unilateral nigrostriatal

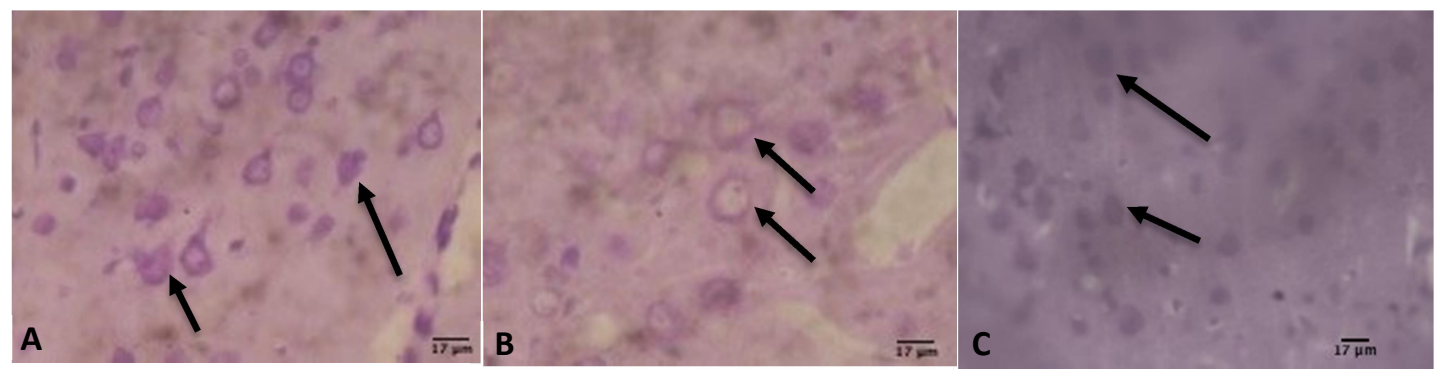

Fig. 11: Photomicrograph of motor cortex Nissl stain $5 \mu$ : (A) Baseline; black arrow showing normal neuron (B) Cassava fed rat; black arrow showing cytoplasmic vacuolation. (C) Post cassava fed rat; black arrow showing less vacoulations

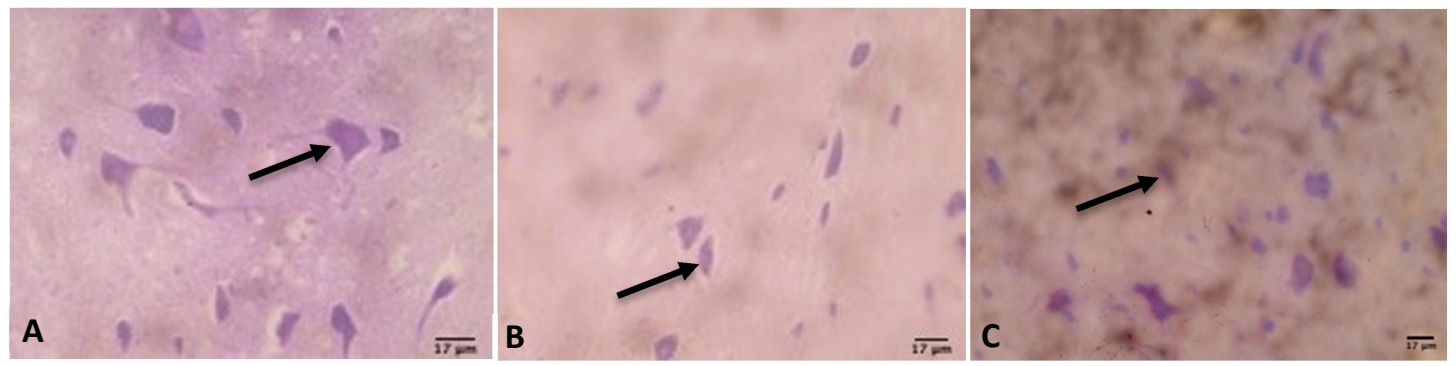

Fig. 12: Photomicrographs of the ventral horn $(5 \mu)$ of spinal cord for Nissl stain: $(A)$ Baseline showing more alpha motor neurons in motor neuron pool $(x 100)$. (B) Cassava toxicity rat showing fewer motor neuron in motor neuron pool (x100). Black arrow showing large motor neuron.
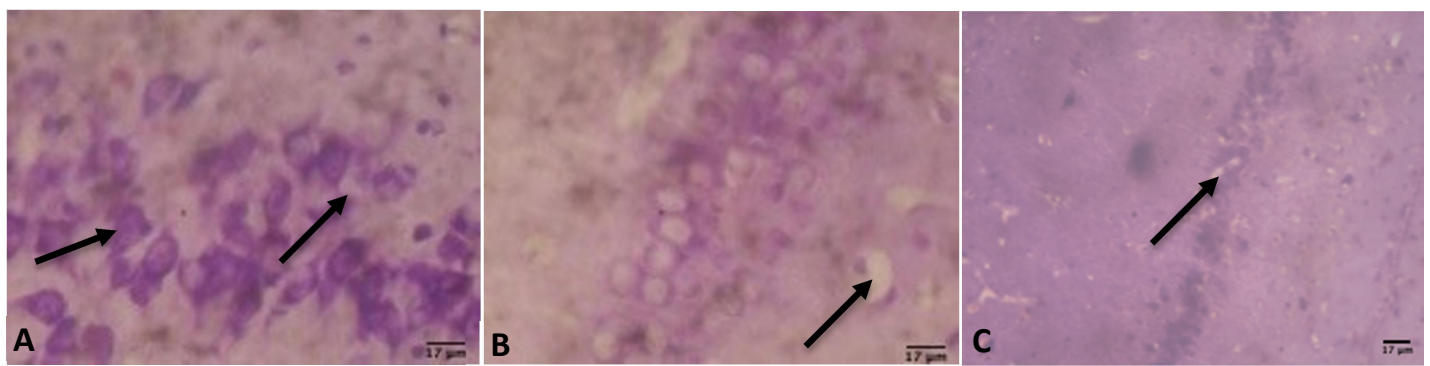

Fig. 13: Photomicrographs of the hippocampus for Nissl stain: (A) Baseline. (B) Cassava fed rat. Black arrow showing vacuolation $(C)$ Post cassava rat. Black arrow showing less vacoulation 
dopamine (DA) depleted rats. Their report showed that unilateral nigrostriatal DA depleted rats displayed impairment in skilled reaching not only with the paw contralateral to the lesion but also with the paw ipsilateral to the lesion. On the expectation that deficits should be seen only on the contralateral side of the body, Montoya et al. (1990) suggested that the ipsilateral impairment may be secondary to "a nonspecific postural deviation rather than a specific impairment in skilled motor use of the ipsilateral limb". Similarly, Miklyaeva et al. (1994) ascribed the ipsilateral impairment to compensatory postural adjustments for postural abnormalities in which the dopaminedepleted rats relied excessively on the ipsilateral hind limb for support and movement. Our findings suggest that cassava consumption produces an acute period of toxicity followed by a toxicity recovery period. The swollen limbs observed may have affected their level of performance in the reach-grasp task.

We confirm that konzo induced rats fed with bitter cassava have an impairment in their success in retrieving food pellets using the paws, in which a success was counted only when the food was retrieved on a single reach. A video analysis of the reaching movements using a tracker software indicated that the rats fed with cassava showed uncoordinated movement (Fig. 9B), with variable trajectories from trial-to-trial. The rats made several attempts before getting the pellet. This is an indication of aiming impairment. The rats displayed impairment in supinating the paw to bring food to the mouth. This was seen in their trajectory as shown in Figure 9C. Rather than supinate, the paw was adducted across the slit so that the mouth contacted the upper surface of the wrist/paw. Food was lost because the paw was eventually placed on the floor of the reaching apparatus. The finding that konzo induced rat produced an impairment is consistent with findings in human konzo patients (Tylleskar et al. 1993).

Looking at the control rat, the average velocity was higher than the experimental rats, and the momentum at take-off was also higher. This indicates that there was no impairment in these rats. It is expected that the velocity should increase from the beginning after the initial velocity and decreases as paws approach the targets. The increase in velocity in the impaired rats as the paw reaches the target is responsible for overreaching, under reaching and miss of target observed in this group (Fig 4B \& C).

As earlier stated that velocity should increase initially and gradually; decrease as it approaches the target, the post cassava rats showed a level of normalcy since its initial velocity was high and gradually reduced. The animal did not support its reaching with the other paw or any other body part (Fig. 6). This shows that there was a level of recovery or healing of the corticospinal tract which was previously reported

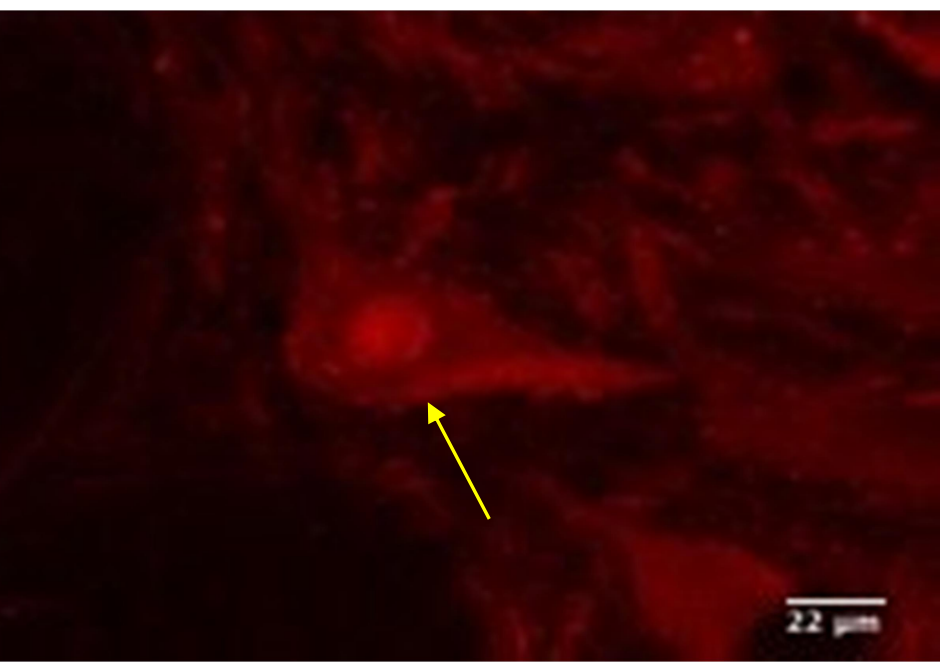

Fig..14: Confocal micrograph stained for the motoneuron marker Choline acetyltransferase (ChAT) of rat after 5 weeks of cassava consumption. Arrow showing presence of Coutons (bright puncta of label) and some numerous ChAT+ ocesses.

to be impaired by the toxicity of cyanide. Our plan was also to explore if the underlying movement impairments after cassava consumption reflects a disruption in central or peripheral myelin.

For the nervous system to function properly, timely delivery of information and precise regulation of neuronal conduction velocity is vital for correct exertion of motor skills, sensory integration and cognitive functions (Seidl 2014). The difference observed in movement velocity in this experiment make us to think that there may be changes in the myelination of axons. It is likely the cyanide toxicity of the bitter cassava may have damaged the myelin sheath or the glia cells producing the myelination. The speed of signal propagation and conduction in neuron is altered by impairments of motor, sensory and possibly cognitive skills (Compston and Coles 2002).

In the ventral horn of spinal cord for Nissl stain, there were more motor neurons in motor neuron pool when compared with cassava toxicity rat with fewer motor neurons in motor neuron pool. This indicates the occurrence of neuropathological changes due to cassava toxicity. Our study has demonstrated and showed evidence of cortical damage due to disruption of pyramidal cells observed in the hippocampus as shown in Figure 13b, but we could not conclude that there was cognitive impairment because neurobehaviour test for cognition was not carried out. We hope to carry out study on cognition and memory in the future. The C-boutons observed in Figure 14 plays a role in cholinergic modulation of 
motor neurons for the locomotor compensation of severe motor neuron loss.

Konzo may be similar to demyelination diseases that degrade the myelin coating on cells e.g. GuillainBarre syndrome and multiple sclerosis. Guillain-Barre syndrome is the destruction of Schwann cells (in the peripheral nervous system), while multiple sclerosis is caused by a loss of oligodendrocytes (in the brain and spinal cord). These disorders have different causes and presentations, but both involve muscle weakness and numbness or tingling as observed also in konzo cassava neurotoxic rat. These symptoms occur because the nerves aren't sending information the right way. When the myelin coating of nerves degenerates, the signals are either diminished or completely destroyed. If the nerves are afferent (sensory) fibres, the destruction of myelin leads to numbness or tingling, because sensations aren't traveling the way they should. We did not carry out sensory test for numbness in this study. When efferent (motor) neurons are demyelinated, this can lead to weakness because the brain is expending a lot of energy, but is still unable to actually move the affected limbs. In this study we observed weakness of limbs, and oedema on the limbs in the cassava toxicity induced rat (Fig 6).

\section{Conclusion}

Our study showed that cassava consumption disrupts skilled movement in rats, which correlated with the neuropathological changes in the motor cortex and the ventral horn of the spinal cord that may underlie movement impairments. There were also neuropathological changes in the hippocampal formation, indicating that cassava consumption in rat models shows features of konzo in humans. This study of rodent model of konzo disease provides significant insight into the study of this epidemic disease in Africa and can be extrapolated to humans.

\section{Conflict of Interest}

None declared.

\section{REFERENCES}

Ballerman, M., Metz, G.A.S., McKenna, J.E., Klassen, F. and Whishaw, I.Q. (2001) The pasta matrix reaching task: a simple test for measuring skilled reaching distance, direction and dexterity in rats. J Neurosci Methods. 106:39-45.

Biernaskie, J. and Corbett, D. (2011) Enriched rehabilitative training promotes improved forelimb motor function and enhanced dendritic growth after focal ischemic injury. J Nuerosci. 21: 5272-5280.

Bradbury, J.H. (2004) Konzo counts. CCDN news. 3(1).

Compston, A. and Coles, A. (2002) Review multiple sclerosis. Lancet. 359(9313):1221-1231.
Dollet, J.M., Beck, B., Villaume, C., Max, J.P. and Debry, G. (1985) Progressive adaption of the endocrine pancreas during long term protein deficiency in rats: Effects on blood glucose homeostasis on pancreas insulin, glucagon and somatostatin concentrations. J Nutr. 155:1581-1588.

Farr, T.F. and Whishaw, I.Q. (2002) Quantitative and qualitative impairments in in skilled reaching in mouse after a focal motor cortex stroke. Stroke. 33(7):1869-1875.

Hermer-Vazquez, L., Hermer-Vazquez, R. and Chapin, J.K. (2007) The reach-to-grasp food task for rats: Arare of modularity in animal behavior. Behav Brain Res. 177(2):322-328.

Howlett, W.P., Brubaker, G.R., Mlingi, M, and Rosling, H. (1990) Konzo, an epidemic upper motor neuron disease studied in Tanzania. Brain. 113:223235.

Kolb, B., Cioe, J. and Whishaw, I.Q. (2000) Is there an optimal age from recovery motor cortex lesion? Behavioural and anatomical consequences of unilateral cortex lesion in perinatal, infant, and adult rats. Restorative Neurol Neurosci. 17:61

McKenna, J.E. and Whishaw, I.Q. (1999) Complete compensation in skilled reaching success with associated impairment in limb synergies, after dorsal column lesion in rat. J Neurosci. 19:1885-1894.

Miklyaeva, E.I, and Whishaw, I.Q. (1996) Hemi Parkinson analogue rats display active support in good limbs versus passive support in bad limbs on a skilled reaching task of variable height. Behav Neurosci. 110(1):117-125. doi:10.1037//07357044.11 0.1.117.

Miklyaeva, E.I., Castañ eda, E. and Whishaw, I.Q. (1994) Skilled reaching deficits in unilateral dopamine-depleted rats: impairments in movement and posture and compensatory adjustments. Journal of Neuroscience. 14:7148-7158.

Montoya, C.P., Astell, S. and Dunnett, S.B. (1990) Effects of nigral and striatal grafts on skilled forelimb use in the rat. Prog Brain Res. 82:459-466.

Ngudi, D.D., Kuo, Y.-H., Van Montagu, M. and Lambien, F. (2012) Research on motor neuron diseases, konzo and neurolathyrism: Trends from 1990 to 2010. PLoS Neglected Trop Dis. 6(7): e1759.doi:10.1371/journal. pntd. 0001759.

Nzwalo, H. and Cliff, J.C. (2011) Konzo: from poverty, cassava and cyanogen intake to toxiconutritional neurological disease. PLOS Neglected Trop Dis. 5(6): e1051.

Okitolonda, W., Brichard, S.M., Pottier, A.M. and Henquin, J.C. (1988) Influence of low and high protein diet on glucose homeostasis in the rats. $\mathrm{Br} \mathrm{J}$ Nutr. 60:509-516.

Sanguanpong, V., Chotineeranatm, S., Piyachomk wan, K., Oates, C.G., Chinachoti, P. and Sriroth, K. (2003) Preparation and structural properties of smallparticle cassava starch. J Sci Food Agric. 83(8):760768. doi:10.1002/jsfa.1374. 
Seidl, A.H. (2014) Regulation of Conduction Time along Axons. Neurosci. 2014:126-134.

Tonukari, J.N. (2004) Cassava and future of starch. Electron J Biotech 7(1):1-8.

Tylleskar T., Howlett, W.P., Rwiza, H.T., Aquilonius, S.M., Stalberg, E., Linden, B., et al. (1993) Konzo: a distinct disease entity with selective upper motor neuron damage. J Neurol Neurosurg Psychiatry. 56:638-645.

U.S.E.P.A. (1988) Recommendations for and Documentation of Biological Values for Use in Risk Assessment. United States Environmental Protection Agency. EPA/600/6-87/008: 1-19

Wang, Z.Y., Lian, H., Zhou, L., Zhang, Y.M., Cai, Q.Q., Zheng, L.F., et al. (2016) Altered expression of D1 and D2 dopamine receptors in vagal neurons innervating the gastric muscularis externa in a
Parkinson's disease rat model. J Parkinsons Dis. 6:317-323. doi: 10.3233/JPD- 160817

Whishaw, I.Q. and Pellis, S.M. (1990) The structure of skilled forelimb reaching in the rat: a proximally driven movement with a single distal rotatory component. Behav Brain Res. 7 41(1):49-59.

Whishaw, I.Q. (2000) Loss of the innate cortical engram for action patterns used in skilled reaching and the development of behavioural compensation following motor cortex lesions in the rat. Neuropharmacol. 39:788-805.

Whishaw, I.Q. and Coles, B.L. (1996) Varieties of paw and digit movement during spontaneous food handling in rats: postures, bimanual coordination, preference and the effect of forelimb cortex lesions. Behav Brain Res. 77(1996):135-148.

Cite as David LK, Paul CW, Chigeru P, Martin JH (2021) Rodent experimental model of konzo: Characterization of motor impairment and neurodegeneration after cassava neurotoxicity in the rat. Nig. J. Neurosci. 12(1):1-13. http://doi.org/10.47081/njn2021.12.1/001 